\title{
Dual Frequency Comb Photonic Analog to Digital Conversion
}

\author{
Callum Deakin \\ Department of Electronic \\ and Electrical Engineering, \\ University College London, \\ London, U.K. \\ callum.deakin.17@ucl.ac.uk
}

\author{
Temitope Odedeyi \\ Department of Electronic \\ and Electrical Engineering, \\ University College London, \\ London, U.K. \\ temitope.odedeyi.13@ucl.ac.uk
}

\author{
Zhixin Liu \\ Department of Electronic \\ and Electrical Engineering, \\ University College London, \\ London, U.K. \\ zhixin.liu@ucl.ac.uk
}

\begin{abstract}
We demonstrate a performance improvement of dual-comb based optical analog-to-digital conversion methods. Through improved optical front-end design, we obtain 4.5 bits ENOB up to $12.5 \mathrm{GHz}$, representing $10 \mathrm{~dB}$ SINAD enhancement compared to previous results.

Index Terms-analog-to-digital converter, optical frequency comb, signal processing
\end{abstract}

\section{INTRODUCTION}

Analog to digital converters (ADCs) are the fundamental technology that allows the capture and analysis of signals across all scientific and engineering disciplines. ADCs are a key component of digital communications links, where the use of high order modulation formats (e.g. $50 \mathrm{GBd}$ 64-QAM) necessitates ADCs that can operate with both high resolution and bandwidth. However, the realization of high speed, high resolution ADCs is extremely challenging. The bandwidth and resolution of conventional electronic ADCs exhibit a tradeoff, mostly due to the strict requirements on sampling clock jitter at high frequencies. At especially high sampling frequencies $(>10 \mathrm{GHz})$, the resolution is further degraded by the speed limitations of the component transistors which results in comparator ambiguity [1].

To combat this tradeoff, high speed ADC designers have developed time and frequency interleaving techniques that utilize a bank of low speed ADCs in parallel to achieve a high aggregate sampling rate whilst minimising the resolution penalty. Electronic time interleaving is the predominant technique to achieve high sampling rates in commercial ADCs [2]. Compared to time-interleaving methods, frequency interleaving methods are less susceptible to channel mismatch errors, and so can potentially overcome the timing jitter and comparator ambiguity limits. However, they are deployed less frequently since they necessitate the design of highly accurate analog electronic filter banks and the synthesis of low noise local oscillators [3]. We previously demonstrated a dual-frequency comb based method for performing frequency interleaving in the optical domain, eliminating the need for high frequency electronic filters or oscillators that has limited the implementation of frequency interleaving ADCs [4].

Funding: EPSRC grant (EP/R041792/1), EPSRC programme grant TRANSNET (EP/R035342/1), Royal Society (PIF/R1/180001).
However, the achieved performance was limited to due to the non-optimized receiver front end design.

In this paper, we demonstrate a $10 \mathrm{~dB}$ increase of the signal to noise and distortion ratio (SINAD) performance with improved receiver front end design. Based on the IEEE standard [5], our proof-of-concept experiment showed an average effective number of bits (ENOB) resolution of 4.5 bits, across $12.5 \mathrm{GHz}$ bandwidth. While this is a modest performance compared to the state-of-the-art electronic time-interleaving ADCs, it demonstrates the possibility of achieving significant performance improvement in dual-comb based ADCs. Furthermore, the potential for further performance scaling makes this an attractive technique, providing an alternative to ADCs based on the electronic time-interleaving technique.

\section{CONCEPT AND EXPERIMENT}

The concept of the proposed photonic ADC is shown in Fig. 1. Two frequency combs of spacing $f_{\text {sig }}$ ('signal comb') and $f_{\text {ref }}=f_{\text {sig }}+\Delta f$ ('reference comb') with $N$ comb lines are generated from the same seed laser $(5 \mathrm{kHz}$ linewidth) to ensure mutual coherence. The signal comb is modulated by a Mach-Zehnder modulator (MZM) biased at the null with the input analog signal under test, resulting in every comb line containing the full signal bandwidth. After recombination with the reference comb, the resulting optical signal is demultiplexed (e.g. via an arrayed waveguide grating) into $N$ channels, resulting in each channel containing a full copy of the analog input signal along with a local oscillator offset by $n \Delta f$ from the input signal baseband.

When detected by a photo receiver, beating between the signal and reference comb in the $n$-th channel will generate a baseband signal centered at $n \Delta f$ from the baseband of the original input signal. With an appropriate anti-aliasing filter, a bank of low speed high resolution ADCs can then be used to digitize a $n \Delta f$ spectral slice of the original signal, which can then be reconstructed digitally and achieve high resolution digitization of the full bandwidth signal. In this way, an input signal of bandwidth $B$ can be fully digitised at the resolution of the sub-ADCs provided that $f_{s i g}>2 B$ and that sufficient comb lines can be generated, i.e. $N \Delta f>B$. 


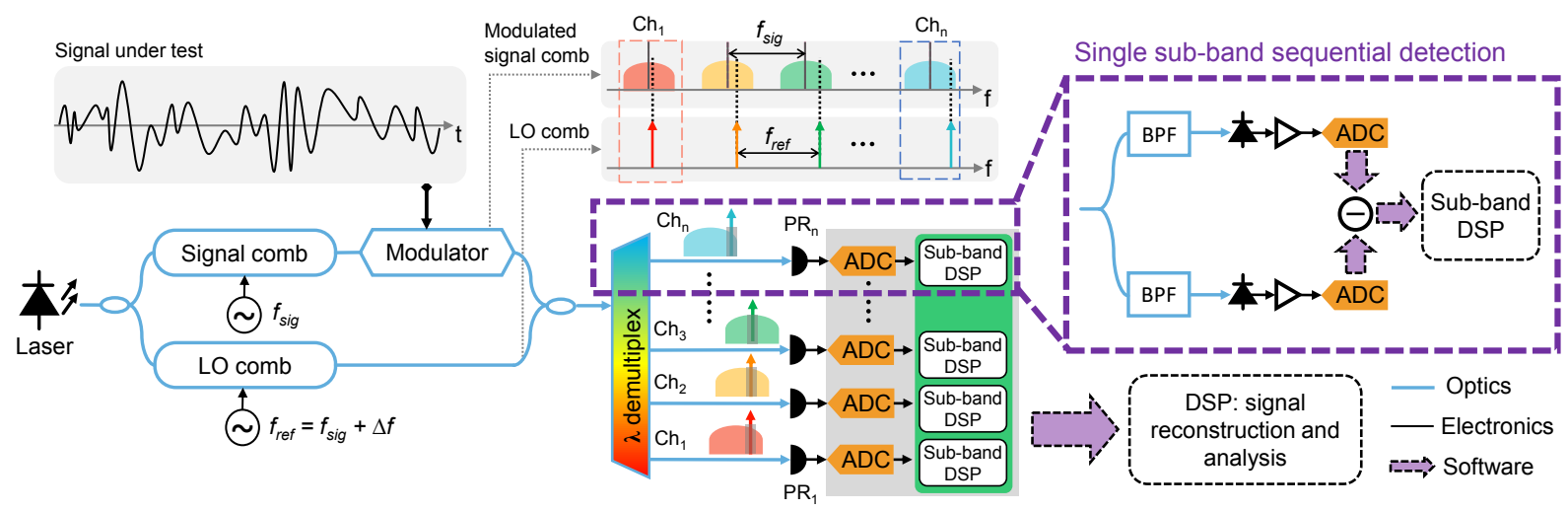

Fig. 1. Dual comb analog to digital converter concept. In this paper, we demonstrate the concept by performing sequential balanced detection of each sub-band, as shown in inset.
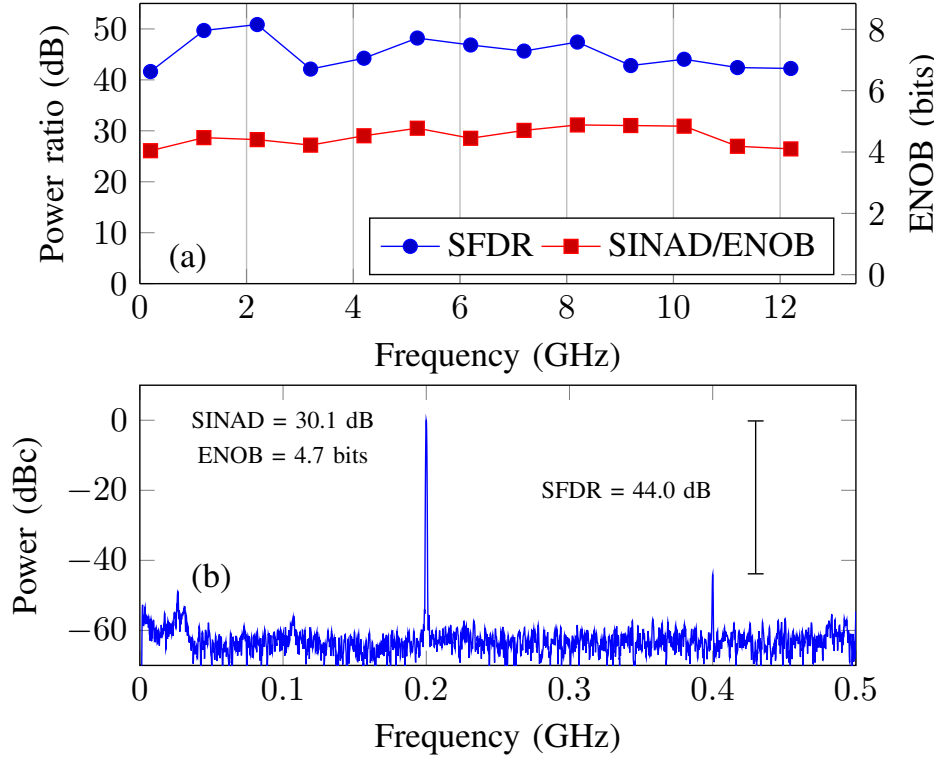

Fig. 2. (a) Measured SFDR and SINAD up to $12.5 \mathrm{GHz}$, with ENOB = (SINAD - 1.76)/6.02. (b) An example fast fourier transform showing a $10.2 \mathrm{GHz}$ signal detected in the $9.5-10.5 \mathrm{GHz}$ sub-band. All values are calculated over a $1 \mathrm{GHz}$ range after digital filtering with an average of four 16384-point FFTs, corresponding to a resolution bandwidth of $61 \mathrm{kHz}$.

As a proof of concept experiment, we perform sequential balanced detection of each sub-band as shown in the inset of Fig. 1. This allows for the detection of sub-band limited signals in order to test the potential performance of such a system without the significant investment of acquiring sufficient ADCs to detect a full bandwidth signal. Note that full coherent detection of each sub-band is needed to detect a full bandwidth arbitrary signal as well as channel restitching DSP, the techniques for which are well established [6].

In this experiment, frequency combs of 25 comb lines and with spacing $f_{\text {sig }}=26 \mathrm{GHz}$ and $f_{\text {sig }}=27 \mathrm{GHz}$ were generated using cascaded intensity and phase modulators. The signal comb was modulated with a sinusoidal input wave, based on the IEEE ADC testing standard, via a Mach-Zehnder modulator biased at the null, with the driving voltage kept less than $0.3 \mathrm{~V}_{\pi}$ to ensure a linear mapping from the electronic to optical domain. A pair of $26 \mathrm{GHz}$ tunable optical bandpass filters were used to select the target sub-band by setting the center wavelength to the appropriate comb line. The signal to noise and distortion ratio (SINAD), spurious free dymanic range (SFDR) and effective number of bits (ENOB) is shown in Fig. 2 for a range of input frequencies covering 0-12.5 GHz, along with an example spectrum in the 9.5-10.5 sub-band. We achieve an average 4.5 bits ENOB across 0$12.5 \mathrm{GHz}$, with a worst case result of 4.1 bits in the 11.5$12.5 \mathrm{GHz}$ sub-band. Overall performance is limited by the strong local osclillator driving the transimpedence amplifier into nonlinear operation, which could be improved by using balanced detection techniques.

\section{CONCLUSION}

We demonstrate an improved result for dual frequency comb based photonic ADCs, achieving 4.5 bits average ENOB for $12.5 \mathrm{GHz}$ bandwidth in sinusoidal wave testing. Potential future improvements to the receiver side analog electronics could significantly increase performance and offer a route to high bandwidth high resolution ADCs based on dual comb photonic frequency interleaving.

\section{REFERENCES}

[1] R. H. Walden, "Analog-to-digital converter survey and analysis," IEEE Journal on selected areas in communications, vol. 17, no. 4, pp. 539$550,1999$.

[2] T. Drenski and J. C. Rasmussen, "ADC \& DAC - Technology trends and steps to overcome current limitations," in 2018 OFC, p. M2C.1, 2018.

[3] S. R. Velazquez, Hybrid filter banks for analog/digital conversion. $\mathrm{PhD}$ thesis, Massachusetts Institute of Technology, 1997.

[4] C. Deakin and Z. Liu, "Dual frequency comb assisted analog-to-digital conversion," Optics Letters, vol. 45, pp. 173-176, Jan. 2020.

[5] IEEE, "IEEE standard for terminology and test methods for analog-todigital converters," IEEE Std 1241-2010 (Revision of IEEE Std 12412000), pp. 1-139, Jan 2011.

[6] L. Guo, S. Tian, Z. Wang, K. Yang, and L. Qiu, "Analysis of Channel Mismatch Errors in Frequency-Interleaved ADC System," Circuits, Systems, and Signal Processing, vol. 33, pp. 3697-3712, Dec. 2014. 